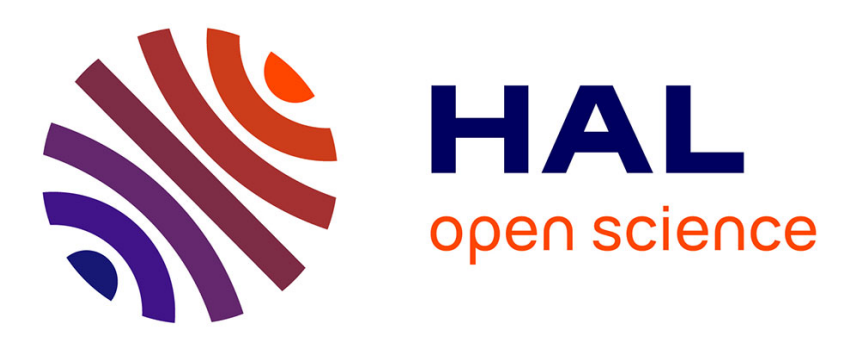

\title{
Calcul de la sensibilité et de la constante de temps d'une thermopile à éléments en couches minces et structure radiale
}

\author{
A. Septier, C. Machet
}

\section{- To cite this version:}

A. Septier, C. Machet. Calcul de la sensibilité et de la constante de temps d'une thermopile à éléments en couches minces et structure radiale. Revue de Physique Appliquée, 1983, 18 (8), pp.507-514. 10.1051/rphysap:01983001808050700 . jpa-00245112

HAL Id: jpa-00245112

https://hal.science/jpa-00245112

Submitted on 1 Jan 1983

HAL is a multi-disciplinary open access archive for the deposit and dissemination of scientific research documents, whether they are published or not. The documents may come from teaching and research institutions in France or abroad, or from public or private research centers.
L'archive ouverte pluridisciplinaire $\mathbf{H A L}$, est destinée au dépôt et à la diffusion de documents scientifiques de niveau recherche, publiés ou non, émanant des établissements d'enseignement et de recherche français ou étrangers, des laboratoires publics ou privés. 


\title{
Calcul de la sensibilité et de la constante de temps d'une thermopile à éléments en couches minces et structure radiale
}

\author{
A. Septier et C. Machet \\ Conservatoire National des Arts et Métiers, ERA 660, \\ Laboratoire de Physique du Vide et des Composants Electroniques, \\ 292, rue Saint-Martin, 75141 Paris Cedex 03, France
}

(Reçu le 9 décembre 1982, révisé le 25 mars 1983, accepté le 12 avril 1983)

\begin{abstract}
Résumé. - A l'aide d'un modèle simple, on calcule de façon approchée la sensibilité et la constante de temps de thermopiles à éléments semiconducteurs en films minces déposés sur un substrat isolant mince, selon une disposition radiale. Le récepteur est un disque métallique central, couvert de noir d'or. Le flux rayonné est plus important que le flux qui s'écoule par conduction. La convection est négligée, l'ensemble étant sous vide. Les résultats donnés par le calcul sont en très bon accord avec les résultats expérimentaux. On obtient, pour la sensibilité au flux $S_{\phi}=68,5 \mathrm{~V} \mathrm{~W}^{-1}$, et pour la constante de temps $\tau=0,76 \mathrm{~s}$.
\end{abstract}

\begin{abstract}
Using a simple model, sensitivity and time constant of a thermopile made of semiconducting thin thermoelements with a radial symmetry and operating in a vacuum, have been calculated. The radiation flux, collected by a metal disc covered with a thick layer of gold black, flows to the heat reservoir at constant temperature $T_{0}$ surrounding the receiver by conduction and thermal radiation. A flux sensitivity $S_{\phi}=68.5 \mathrm{~V} \mathrm{~W}^{-1}$ and a time constant $\tau=0.76 \mathrm{~s}$ have been obtained, in good agreement with experiments.
\end{abstract}

\section{Introduction.}

Un radiomètre à thermopile destiné à la métrologie de faibles flux lumineux constitue un générateur de tension. Sa résistance interne peut être élevée, contrairement au cas des thermopiles utilisées en générateurs de courants. Il est donc possible de réaliser les éléments actifs des thermocouples sous forme de films minces déposés sur un support approprié, à la fois isolant électrique et thermique. Cette technique se prête bien à la miniaturisation des thermocouples. Les éléments actifs peuvent être métalliques ( $\mathrm{Au}-\mathrm{Ni}$ [1] ou $\mathrm{Sb}-\mathrm{Bi}$ [2]) ou semiconducteurs $n$ et $\mathrm{p}$ [3-5].

Parmi les réalisations récentes de thermopiles en films minces, on peut distinguer deux types de structures : les éléments actifs, déposés sous forme de rubans, connectés en série, peuvent être disposés linéairement suivant des segments parallèles $[1,3]$, ou bien radialement [2,5]. Ce dernier dispositif comporte un disque métallique central recevant le flux à mesurer, isolé thermiquement au centre d'un orifice circulaire percé dans un corps massif à température constante. Il a déjà été étudié du point de vue théorique, mais avec des thermocouples à fils $[6,7]$.
Nous avons tenté d'évaluer la sensibilité en régime continu et la constante de temps d'une thermopile à éléments radiaux réalisés en films minces semiconducteurs, et comparé résultats théoriques et résultats expérimentaux obtenus sur une structure très proche du modèle utilisé pour les calculs.

\section{Structure de la thermopile.}

Une membrane mince de mylar, d'épaisseur $3 \mu \mathrm{m}$, est tendue sur un orifice circulaire de diamètre $2 r_{2}=6 \mathrm{~mm}$ percé dans un bloc de cuivre, servant de source froide à la température $T_{0}$. A l'aide de masques, on évapore sur la face supérieure du film de mylar 45 éléments de semiconducteur $\mathrm{n}$, puis 45 éléments de semiconducteur $p$ formant 45 thermocouples (Fig. 1a). Les soudures chaudes et froides sont situées respectivement sur des cercles moyens de diamètre $4 \mathrm{~mm}$ et $8 \mathrm{~mm}$. La largeur des barreaux de matériau semiconducteur est de $65 \mu \mathrm{m}$; leur épaisseur de $0,3 \mu \mathrm{m}$. Les soudures froides sont isolées du support de cuivre par l'épaisseur du film de mylar (Fig. 1b). On considérera que la conductivité 


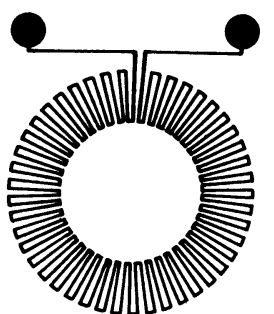

a)

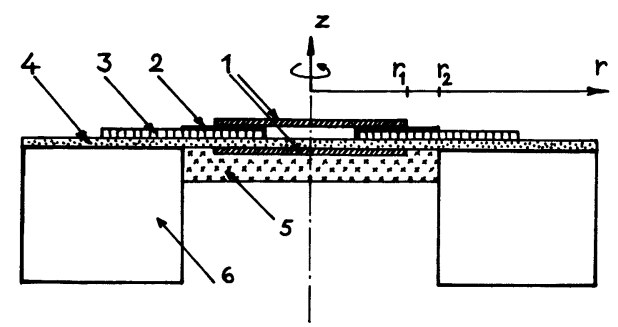

b)

Fig. 1. - Structure de la thermopile. (a) Disposition radiale des thermocouples. (b) Coupe schématique de la thermopile. 1 : couches d'or; 2 : isolant très mince; 3 : thermoéléments ; 4 : support isolant (mylar); 5 : noir d'or ; 6 : support massif cuivre (source froide).

[Structure of the thermopile. (a) Plan view of the radial thermocouples. (b) Schematic cross-section. 1 : gold layers; 2 : very thin insulator; $3:$ thermoelements; 4 : insulating film (mylar); 5 : gold black layer; 6 : copper support (heat reservoir).]

tranșversale de ce film est suffisante pour que la température des soudures froides soit constante et égale à $T_{0}$.

Un disque métallique de diamètre $2 r_{1}=5 \mathrm{~mm}$, constituant le récepteur, est évaporé sur la face inférieure du film de mylar, afin de permettre la thermalisation de la zone centrale. Une couche épaisse de noir d'or est ensuite déposée sur le disque central pour absorber le flux lumineux incident. Le coefficient d'absorption du noir d'or est très voisin de 1 dans le visible et le proche infrarouge. Pour des raisons technologiques, il est pratiquement impossible de recouvrir de façon homogène le disque métallique central de diamètre $2 r_{1}$. Le noir d'or occupe donc la zone de diamètre $2 r_{2}$ et est en contact avec le support de cuivre, mais sa conductivité thermique est suffisamment faible [8] pour ne pas perturber le fonctionnement de la thermopile.

On suppose en outre qu'un second disque métallique parfaitement réfléchissant de $5 \mathrm{~mm}$ de diamètre est déposé au-dessus des soudures chaudes, préalablement recouvertes d'une très mince couche électriquement isolante ( $\mathrm{SiO}$ par exemple).

Pour l'analyse de l'écoulement thermique dans une telle thermopile, nous adopterons un modèle simplifié

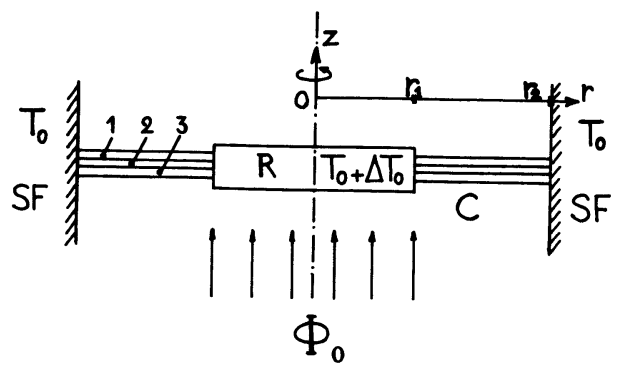

a)

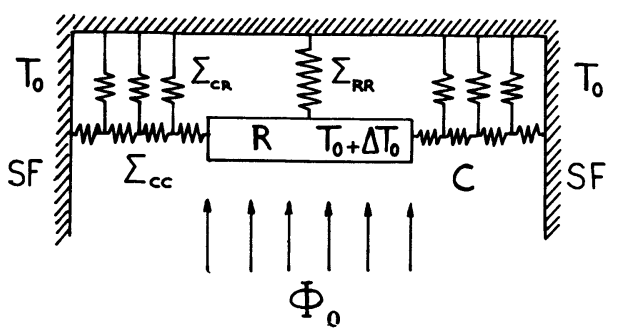

b)

Fig. 2. - Modèle simplifié en forme de disque. (a) Coupe de la thermopile. $\mathrm{R}=$ récepteur central isotherme à $T=$ $T_{0}+\Delta T_{0}$, de rayon $r_{1}$. C $=$ couronne à 3 milieux $(1$ : thermoéléments, $2:$ mylar, $3:$ noir d'or). $\mathrm{SF}=$ source froide de rayon intérieur $r_{2}$ à $T=T_{0} . \Phi_{0}=$ flux incident limité à $r<r_{1}$. (b) Conductances thermiques. $\Sigma_{\mathrm{RR}}=$ rayonnement du récepteur. $\Sigma_{\mathrm{CC}}=$ conduction dans la couronne. $\Sigma_{\mathrm{CR}}=$ rayonnement réparti entre $r_{1}$ et $r_{2}$. SF = source froide.

[Simplified disc shaped model. (a) Thermopile cross section. $\mathrm{R}=$ central photon receiver at $T=T_{0}+\Delta T_{0}$, external radius $r_{1} . C=$ layered ring ( $1:$ thermoelements, $2:$ mylar, 3 : gold black). $\mathrm{SF}=$ heat reservoir at $T=T_{0}$, internal radius $r_{2} . \Phi_{0}=$ incident radiation flux $\left(0<r<r_{1}\right)$. (b) Thermal conductances. $\Sigma_{\mathrm{RR}}=$ receiver thermal radiation. $\Sigma_{\mathrm{CC}}=$ thermal conduction from receiver to external body. $\Sigma_{\mathrm{CR}}=$ thermal radiation from the external ring. $\mathrm{SF}=$ heat reservoir at $T=T_{0}$.

à symétrie de révolution autour d'un axe $\mathrm{O} z$ représenté sur la figure $2 a$. Le récepteur de flux constitué par la région centrale de $5 \mathrm{~mm}$ de diamètre recouverte, sur une face, d'un absorbant parfait et sur l'autre face d'un métal d'émissivité nulle, est relié au support de cuivre par une conductance thermique radiale. Si l'on suppose que la conductivité thermique des disques métalliques centraux est infinie, la température du récepteur, soumis au flux incident $\Phi$ sur toute sa surface $A_{1}=\pi r_{1}^{2}\left(\right.$ avec $\left.r_{1}=2,5 \mathrm{~mm}\right)$ est homogène et égale à $T=T_{0}+\Delta T_{0}\left(\Delta T_{0} \ll T_{0}\right)$. Une partie du flux est rayonnée à partir du récepteur vers l'environnement extérieur, supposé à la température $T_{0}$. Le reste s'écoule radialement vers le support de cuivre, mais avec un rayonnement partiel vers l'enceinte.

La thermopile est supposée placée sous vide ce qui supprime les pertes thermiques par conduction et convection dans le gaz environnant. 


\section{Sensibilité de la thermopile.}

En désignant par $\alpha_{\mathrm{S}}$ le coefficient de Seebeck d'un thermocouple, $N$ le nombre de thermocouples connectés en série, et $\Phi_{0}=E_{0} A_{1}$ le flux incident ( $E_{0}$ est l'éclairement supposé homogène), la sensibilité au flux, exprimée en V par W, est donnée par l'expression :

$$
S_{\phi}=\frac{N \alpha_{S} \Delta T_{0}}{\Phi_{0}}\left(\mathrm{~V} \mathrm{~W}^{-1}\right)
$$

et la sensibilité à l'éclairement par :

$$
S_{\mathrm{E}}=\frac{N \alpha_{\mathrm{S}} \Delta T_{0}}{E_{0}}=S_{\phi} A_{1} \quad\left(\mathrm{~V} \mathrm{~W}^{-1} \mathrm{~m}^{2}\right)
$$

$A_{1}$ est l'aire éclairée du récepteur.

Le récepteur central étant à température constante $T=T_{0}+\Delta T_{0}$ le flux thermique s'écoulant à la périphérie en $r=r_{1}$ s'écrit :

$$
\Phi_{\mathrm{T}}=\Phi_{\mathrm{A}}-\Phi_{\mathrm{R}}
$$

avec

$$
\Phi_{\mathrm{A}}=\mathcal{A} \Phi_{0}
$$

( $\mathcal{A}=$ coefficient d'absorption de la surface) et

$$
\Phi_{\mathrm{R}}=\pi\left(\varepsilon_{1}+\varepsilon_{2}\right) \sigma_{\mathrm{S}} A_{1}\left(T^{4}-T_{0}^{4}\right)
$$

$\left(\sigma_{\mathrm{S}}=\right.$ constante de Stefan égale à $1,80 \times 10^{-8}$ $\left(\mathrm{W} \mathrm{m}{ }^{-2} \mathrm{Sr}^{-1} \mathrm{~K}^{-4}\right.$ ), $\varepsilon_{1}$ et $\varepsilon_{2}=$ émissivités des deux surfaces du récepteur). Lorsqu'on mesure des flux $\Phi_{0}$ faibles, inférieurs à $\Phi_{0}=10^{-3} \mathrm{~W}$ par exemple, l'élévation de température $\Delta T_{0}$ ne dépasse pas 1 degré. Par suite, on peut écrire :

$$
\Phi_{\mathrm{R}}=4 \pi\left(\varepsilon_{1}+\varepsilon_{2}\right) \sigma_{\mathrm{S}} A_{1} \cdot T_{0}^{3} \Delta T_{0} .
$$

Dans notre cas, nous supposerons pour simplifier que pour le noir d'or $A=\varepsilon_{1}=1$, et pour la surface métallisée $\varepsilon_{2}=0$.

Par suite :

$$
\Phi_{\mathrm{T}}=\Phi_{0}-4 \pi \sigma_{\mathrm{S}} A_{1} T_{0}^{3} \Delta T_{0} .
$$

L'écoulement vers la source froide du flux thermique $\Phi_{T}$ s'effectue d'une part à travers la conductance thermique radiale constituée par la membrane de mylar, les barreaux semiconducteurs et le noir d'or, et d'autre part par rayonnement à partir de ces mêmes éléments.

- Pour un élément de surface d'aire $A$ à la température $T_{0}+\Delta T$, la puissance rayonnée s'écrit :

$$
P_{\mathrm{R}}=4 \pi \sigma_{\mathrm{S}} T_{0}^{3} A \Delta T=K_{\mathrm{R}} A \Delta T=\Sigma_{\mathrm{R}} \Delta T .
$$

$\Sigma_{\mathrm{R}}$ représente la conductance thermique équivalente au rayonnement. Pour $T_{0}=300 \mathrm{~K}$, on a :

$$
K_{\mathrm{R}}=6,13 \mathrm{~W} \mathrm{~m}^{-2} \mathrm{~K}^{-1} \text {. }
$$

Cette conductance thermique est répartie entre $r_{1}$ et $r_{2}$ sur la face couverte de noir d'or $\left(\varepsilon_{1}=1\right)$ et on aboutit alors au schéma de la figure $2 b$.

- La conductance thermique d'une couronne de largeur $\mathrm{d} r$ située à la distance $r$ de l'axe $\mathrm{O} z$ s'écrit :

$$
\mathrm{d} \Sigma_{\mathrm{C}}=\frac{2 \pi r}{\mathrm{~d} r} \sum_{i} \gamma_{i} e_{i}
$$

où $\gamma_{i}$ représente la conductivité thermique de l'un des matériaux du milieu stratifié composant la couronne située entre $r_{1}$ et $r_{2}$ (éléments actifs, mylar, noir d'or) et $e_{i}$ l'épaisseur de ce matériau. Les barreaux des thermocouples, supposés de largeur identique $l$, peuvent avoir des conductivités différentes $\gamma_{1}$ et $\gamma_{2}$, et des épaisseurs différentes $e_{1}$ et $e_{2}$. Ils ne recouvrent qu'une partie de la surface du mylar et on définira une conductance moyenne "par carré " $\overline{\gamma_{\text {th }} e_{\text {th }}}$ sur la circonférence moyenne de rayon $\left(r_{1}+r_{2}\right) / 2$ :

$\overline{\gamma_{\text {th }} e_{\mathrm{th}}}=K\left(\gamma_{1} e_{1}+\gamma_{2} e_{2}\right)$ avec $K=N l / \pi\left(r_{1}+r_{2}\right)$.

- Dans le cas simple où on néglige les pertes de flux par rayonnement entre $r_{1}$ et $r_{2}$, on peut calculer simplement la conductance thermique $\Sigma_{\mathrm{CC}}$ de li couronne, et l'écart de température $\Delta T_{0}$

$$
\Delta T_{0}=\frac{\Phi T}{\Sigma_{\mathrm{CC}}}
$$

et :

$$
\frac{1}{\Sigma_{\mathrm{CC}}}=\frac{1}{2 \pi \sum_{i} \gamma_{i} e_{i}} \int_{r_{1}}^{r_{2}} \frac{\mathrm{d} r}{r}
$$

soit :

$$
\Sigma_{\mathrm{CC}}=\frac{2 \pi \sum_{i} \gamma_{i} e_{i}}{\log r_{2} / r_{1}} .
$$

Le flux thermique circulant dans $\Sigma_{\mathrm{CC}}$ est

$$
\Phi_{\mathrm{R}}=\Phi_{0}-K_{\mathrm{R}}\left(\pi r_{1}^{2}\right) \Delta T_{0} .
$$

D’où :

$$
\begin{aligned}
\Delta T_{0} & =\frac{\Phi_{0}-K_{\mathrm{R}} A_{1} \Delta T_{0}}{\Sigma_{\mathrm{CC}}} \quad\left(A_{1}=\pi r_{1}^{2}\right) \\
\Delta T_{0} & =\frac{\Phi_{0}}{\Sigma_{\mathrm{CC}}+K_{\mathrm{R}} A_{1}}=\frac{\Phi_{0}}{\Sigma_{\mathrm{CC}}+\Sigma_{\mathrm{RR}}} .
\end{aligned}
$$

$\Sigma_{\mathrm{RR}}$ est la conductance de rayonnement du récepteur central.

Finalement :

$$
S_{\phi}=\frac{N \alpha_{\mathrm{S}}}{\Sigma_{\mathrm{CC}}+\Sigma_{\mathrm{RR}}}\left(\mathrm{V} \mathrm{W}^{-1}\right) .
$$

- Si le rayonnement à partir de la surface du récepteur est lui-même négligeable, on peut supprimer le 
terme $K_{\mathrm{R}} A_{1}$ au dénominateur. Cela ne sera possible que pour :

$$
\Sigma_{\mathrm{RR}}=K_{\mathrm{R}} A_{1} \ll \Sigma_{\mathrm{CC}}=\frac{2 \pi \sum_{i} \gamma_{i} e_{i}}{\log r_{2} / r_{1}}
$$

c'est-à-dire des matériaux à très forte conductance thermique, ou bien $r_{2} \simeq r_{1}$.

Dans ce cas simple, la répartition de température $T(r)$ entre le récepteur $\left(T=T_{0}+\Delta T_{0}\right.$ en $\left.r=r_{1}\right)$ et la source froide $\left(T=T_{0}\right.$ en $\left.r=r_{2}\right)$ est obtenue en résolvant l'équation de Laplace qui, pour un système à symétrie de révolution sans variation suivant l'axe $\mathrm{O} z$, s'écrit :

$$
\nabla^{2} T(r)=\frac{\mathrm{d}^{2} T(r)}{\mathrm{d} r^{2}}+\frac{1}{r} \frac{\mathrm{d} T(r)}{\mathrm{d} r}=0 .
$$

On obtient :

$$
T(r)-T_{0}=\Delta T_{0}\left(1-\frac{\log r / r_{1}}{\log r_{2} / r_{1}}\right)
$$

Lorsque $\left(r_{2}-r_{1}\right) / r_{1} \ll 1$, la variation de $T(r)$ est pratiquement linéaire (Fig. 3 ).

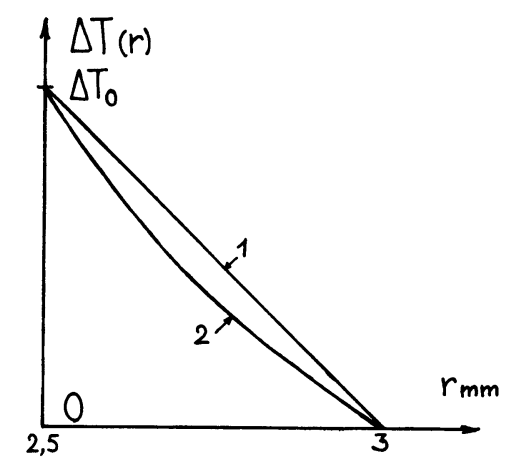

Fig. 3. - Répartition de température entre le récepteur et la source froide $\left(r_{1}<r<r_{2}\right)$. 1: Avec $\Sigma_{\mathrm{CR}}=0 ; 2: \Sigma_{\mathrm{CR}} \neq 0$.

[Temperature variation between receiver and copper heat reservoir. $\left.1: \Sigma_{\mathrm{CR}}=0 ; 2: \Sigma_{\mathrm{CR}} \neq 0.\right]$

- Si l'on s'intéresse maintenant au cas général, pour obtenir la répartition de la température entre $r_{1}$ et $r_{2}$, on doit résoudre une équation de type Poisson $[4,6]$ :

$$
\nabla^{2}(\Delta T(r))-\frac{P(r)}{\sum_{i} \gamma_{i} e_{i}}=0
$$

avec :

$$
P(r)=4 \pi \sigma_{\mathrm{S}} T_{0}^{3} \Delta T(r)=K_{\mathrm{R}} \Delta T(r)
$$

et :

on a donc :

$$
\Delta T(r)=T(r)-T_{0},
$$

$$
\frac{\mathrm{d}^{2}(\Delta T)}{\mathrm{d} r^{2}}+\frac{1}{r} \frac{\mathrm{d}(\Delta T)}{\mathrm{d} r}-\frac{1}{\beta^{2}} \Delta T(r)=0,
$$

avec :

$$
\beta^{2}=\frac{\sum_{i} \gamma_{i} e_{i}}{K_{\mathrm{R}}}
$$

La solution fait intervenir les fonctions de Bessel modifiées $I_{0}(r / \beta)$ et $K_{0}(r / \beta)$ :

$$
\Delta T=A I_{0}\left(\frac{r}{\beta}\right)+B K_{0}\left(\frac{r}{\beta}\right)
$$

On a les conditions aux limites suivantes :

$$
\begin{aligned}
& -\Delta T\left(r_{2}\right)=0 . \\
& -\quad \Delta T\left(r_{1}\right)=\Delta T_{0} .
\end{aligned}
$$

- Flux thermique en $r=r_{1}$.

$$
\begin{aligned}
-2 \pi r_{1}\left(\sum_{i} \gamma_{i} e_{i}\right) & \left(\frac{\mathrm{d}(\Delta T)}{\mathrm{d} r}\right)_{r=r_{1}}= \\
& =-\left(E_{0}-K_{\mathrm{R}} \cdot \Delta T_{0}\right) \pi r_{1}^{2} .
\end{aligned}
$$

On peut calculer les deux constantes $A$ et $B$, à l'aide de (1) et (2) :

$$
A=\frac{-K_{0}\left(r_{2} / \beta\right) \cdot \Delta T_{0}}{Y\left(r_{1}, r_{2}\right)} \quad B=\frac{+I_{0}\left(r_{2} / \beta\right) \Delta T_{0}}{Y\left(r_{1}, r_{2}\right)}
$$

avec :

$$
Y\left(r_{1}, r_{2}\right)=I_{0}\left(\frac{r_{2}}{\beta}\right) K_{0}\left(\frac{r_{1}}{\beta}\right)-I_{0}\left(\frac{r_{1}}{\beta}\right) K_{0}\left(\frac{r_{2}}{\beta}\right) .
$$

On obtient :

$$
\Delta T(r)=\Delta T_{0} \frac{I_{0}\left(r_{2} / \beta\right) K_{0}(r / \beta)-K_{0}\left(r_{2} / \beta\right) I_{0}(r / \beta)}{Y\left(r_{1}, r_{2}\right)}
$$

cette fonction est représentée sur la figure 3.

En utilisant la relation (3) et sachant que :

$$
\frac{\mathrm{d} I_{0}(x)}{\mathrm{d} x}=I_{1}(x) \text { et } \frac{\mathrm{d} K_{0}(x)}{\mathrm{d} x}=-K_{1}(x),
$$

on détermine $\Delta T_{0}$ en fonction de $E_{0}$ :

$$
\begin{aligned}
& \Delta T_{0}=E_{0} \frac{1}{K_{\mathrm{R}}+\frac{2}{r_{1} \beta} \sum_{i} \gamma_{i} e_{i} \cdot F\left(r_{1}, r_{2}\right)} \\
& \Delta T_{0}=E_{0} \frac{1}{K_{\mathrm{R}}+\frac{2}{r_{1}}\left(K_{\mathrm{R}} \cdot \sum_{i} \gamma_{i} e_{i}\right)^{1 / 2} \cdot F\left(r_{1}, r_{2}\right)}
\end{aligned}
$$

avec :

$$
F\left(r_{1}, r_{2}\right)=\frac{K_{0}\left(r_{2} / \beta\right) I_{1}\left(r_{1} / \beta\right)+K_{1}\left(r_{1} / \beta\right) I_{0}\left(r_{2} / \beta\right)}{Y\left(r_{1}, r_{2}\right)}
$$


On en tire :

$$
\begin{aligned}
S_{\mathrm{E}} & =\frac{N \alpha_{\mathrm{S}}}{K_{\mathrm{R}}+\frac{2}{r_{1}}\left(K_{\mathrm{R}} \sum_{i} \gamma_{i} e_{i}\right)^{1 / 2} F\left(r_{1}, r_{2}\right)}, \\
S_{\phi} & =\frac{N \alpha_{\mathrm{S}}}{\pi r_{1}^{2} K_{\mathrm{R}}+2 \pi r_{1}\left(K_{\mathrm{R}} \sum_{i} \gamma_{i} e_{i}\right)^{1 / 2} F\left(r_{1}, r_{2}\right)} \\
& =\frac{N \alpha_{\mathrm{S}}}{\sum_{\mathrm{RR}}+\Sigma_{\mathrm{CT}}} .
\end{aligned}
$$

Le dénominateur de $S_{\phi}$, qui représente la conductance thermique globale du dispositif, est la somme de deux termes : la conductance de rayonnement du disque central $\Sigma_{\mathrm{RR}}=\pi r_{1}^{2} K_{\mathrm{R}}$ et la conductance globale de la couronne $\Sigma_{\mathrm{CT}}=2 \pi r_{1}\left(K_{\mathrm{R}} \sum_{i} \gamma_{i} e_{i}\right)^{1 / 2} \times$ $F\left(r_{1}, r_{2}\right)$.

Le calcul numérique peut être effectué à l'aide des valeurs des différents paramètres donnés dans le tableau I, tirées de [1]

Tableau I.

$\begin{array}{lcccc}\text { Matériaux } & \begin{array}{c}\text { Epaisseur } \\ e_{i}(\mathrm{~m})\end{array} & \begin{array}{c}\text { Conductivité } \\ \text { thermique } \gamma_{i} \\ \left(\mathrm{~W} \mathrm{~m}^{-1} \mathrm{~K}^{-1}\right)\end{array} & \begin{array}{c}\text { Chaleur } \\ \text { massique } C_{i} \\ \left(\mathrm{~J} \mathrm{~kg}^{-1} \mathrm{~K}^{-1}\right)\end{array} & \begin{array}{c}\text { Masse } \\ \text { volumique mi } \\ \left(\mathrm{kg} \mathrm{m}^{-3}\right)\end{array} \\ \text { - } & - & - & - & - \\ \text { et } \mathrm{p}\left(\mathrm{Te}_{2} \mathrm{Bi}_{3}\right) & 0,3 \times 10^{-6} & 1,5 & 2,5 \times 10^{2} & 7,5 \times 10^{3} \\ \text { ylar } & 3 \times 10^{-6} & 1,5 \times 10^{-1} & 1,25 \times 10^{3} & 1,4 \times 10^{3} \\ \text { ir d'or } & 0,2 \times 10^{-6} & 2,9 \times 10^{2} & 1,3 \times 10^{2} & 1,9 \times 10^{4} \\ & 20 \times 10^{-6} & 10^{-2} & 1,3 \times 10^{2} & 3,9 \times 10^{1}\end{array}$

Pour $r_{1}=2,5 \times 10^{-3} \mathrm{~m}$ et $r_{2}=3 \times 10^{-3} \mathrm{~m}$, et un coefficient $\beta$ égal dans notre cas à $\beta=3,62 \times$ $10^{-4} \mathrm{~m}=0,362 \mathrm{~mm}$, on obtient les conductances thermiques globales suivantes :

$$
\begin{aligned}
& \Sigma_{\mathrm{RR}}=1,11 \times 10^{-4} \mathrm{~W} \mathrm{~K}^{-1} ; \\
& \Sigma_{\mathrm{CT}}=0,41 \times 10^{-4} \mathrm{~W} \mathrm{~K}^{-1}
\end{aligned}
$$

soit, au total :

$$
\Sigma_{\mathrm{T}}=1,52 \times 10^{-4} \mathrm{~W} \mathrm{~K}^{-1} .
$$

La conductance globale de la couronne $\Sigma_{\mathrm{CT}}$ est ellemême la somme de deux termes, : une composante $\Sigma_{\mathrm{CR}}$ due au rayonnement et un terme $\Sigma_{\mathrm{CC}}$ dû à la conduction : $\Sigma_{\mathrm{CT}}=\Sigma_{\mathrm{CR}}+\Sigma_{\mathrm{CC}}$ avec (voir plus haut) :

$$
\begin{aligned}
\Sigma_{\mathrm{cC}} & =2 \pi \sum_{i}\left(\gamma_{i} e_{i} / \log \left(r_{2} / r_{1}\right)\right) \\
& =0,27^{\circ} \times 10^{-4} \mathrm{~W} \mathrm{~K}^{-1} \ll \Sigma_{\mathrm{T}} .
\end{aligned}
$$

On voit donc que, pour des éléments actifs semiconducteurs, de faible conductivité thermique, la conductance associée au rayonnement est très supérieure à la conductance thermique de la couronne.

En prenant pour les éléments semiconducteurs en tellurure de bismuth une valeur effectivement mesurée [4] : $\alpha_{\mathrm{S}}=2,5 \times 10^{-4} \mathrm{~V} \mathrm{~K}^{-1}$ on obtient finalement :

$$
S_{\mathrm{E}_{\text {calc }}}=13,7 \mathrm{~V} \mathrm{~cm}^{-2} \mathrm{~W}^{-1},
$$

et :

$$
S_{\phi_{\text {calc }}}=68,5 \mathrm{~V} \mathrm{~W}^{-1} \text {. }
$$

La valeur de $S_{\mathrm{E}}$ obtenue avec cette thermopile est très supérieure à celle des thermopiles étalon utilisées communément en métrologie [9] : $1,26 \mathrm{~V} \mathrm{~W}^{-1} \mathrm{~cm}^{2}$ : - de la thermopile $\mathrm{Sb}-\mathrm{Bi}$ à structure radiale [2] : $3,09 \mathrm{~V} \mathrm{~W}^{-1} \mathrm{~cm}^{2}$,

- des thermopiles RTC commercialisées, à films minces de $\mathrm{Te}_{2} \mathrm{Bi}_{3}$ disposés en segments parallèles [4] : $0,37 \mathrm{VW}^{-1} \mathrm{~cm}^{2}$.

\section{Constante de temps.}

Lorsqu'on interrompt brutalement le flux incident, la tension aux bornes de la thermopile tend vers zéro, mais le refroidissement n'est pas instantané. La connaissance de l'évolution temporelle de $\Delta T(t)$ à la coupure, ou à l'installation du flux incident, conditionne le fonctionnement éventuel de la thermopile avec un flux incident modulé en signaux rectangulaires.

La constante de temps $\tau$ de la thermopile peut être calculée de façon approchée en utilisant l'analogie électrique avec un circuit RC. En désignant par $C_{\mathrm{T}}$ la capacité thermique de la zone centrale recevant le flux lumineux et $\Sigma_{\mathrm{T}}$ la conductance thermique totale vue plus haut, on a :

$$
\tau=C_{\mathrm{T}} / \Sigma_{\mathrm{T}}
$$

avec : $C_{\mathrm{T}}=\pi r_{1}^{2} \sum_{i} e_{i} m_{i} C_{i}\left(m_{i}:\right.$ masse spécifique, $C_{i}$ : chaleur massique).

Dans le modèle utilisé, la zone centrale est composée d'une superposition de 5 milieux : le noir d'or, 2 couches d'or, le mylar et les éléments actifs.

Le calcul numérique utilisant les valeurs du tableau I 
donne, pour la thermopile munie de 2 couches d'or de $0,2 \mu \mathrm{m}$ d'épaisseur :

$$
C_{\mathrm{T}}=1,26 \times 10^{-4} \mathrm{~J} \mathrm{~K}^{-1}
$$

d'où : $\tau_{2_{\text {calc }}}=0,83 \mathrm{~s}$.

En enlevant l'une des couches d'or :

$$
C_{\mathrm{T}}=1,16 \times 10^{-4} \mathrm{~J} . \mathrm{K}^{-1}
$$

on aurait : $\tau_{1_{\text {calc }}}=0,76 \mathrm{~s}$.

La contribution du noir d'or dans $C_{\mathrm{T}}$ est négligeable et celle du semiconducteur très faible. Par contre, le mylar intervient pour plus de $80 \%$, et l'or ( 2 couches) pour $15,5 \%$.

\section{Comparaisons avec l'expérience.}

Plusieurs thermopiles ont été réalisées sur le modèle décrit plus haut (mais avec une seule couche d'or), avec des éléments en tellurure de bismuth $n$ et $p$ [5].

La sensibilité et la constante de temps ont été mesurées à l'Institut National de Métrologie du CNAM. La valeur mèsurée du coefficient Seebeck pour un thermocouple était $\alpha_{\mathrm{s}}=2,5 \times 10^{-4} \mathrm{~V} \mathrm{~K}^{-1}$, et les thermopiles étaient formées de $N=45$ thermocouples connectés en série.

Les mesures ont donné la valeur moyenne suivante pour $S_{\mathrm{E}}$, avec une dispersion de $\pm 7 \%$ :

$$
S_{\mathrm{E}_{\text {exp }}}=13,5 \mathrm{~V} \mathrm{~W}^{-1} \mathrm{~cm}^{-2} \text {, }
$$

c'est-à-dire une valeur très proche de la valeur calculée à l'aide de notre modèle simplifié $-S_{\mathrm{E}_{\mathrm{calc}}}$.

La constante de temps mesurée est en moyenne de :

$$
\tau_{\text {exp }} \simeq 0,82 \mathrm{~s} \text {. }
$$

Le calcul, avec une seule couche d'or, donne $\tau_{\text {calc }}=$ $0,76 \mathrm{~s}$, mais il n'a pas été tenu compte de la capacité thermique $C_{\mathrm{c}}$ de la couronne $r_{1}<r<r_{2}$, portée à une température variant de $T_{0}+\Delta T(0)$ à $T_{0}$.

A partir de la courbe $\Delta T(r)$ obtenu en régime continu, on peut calculer cette capacité thermique additionnelle de la couronne, composée de mylar, matériau SC et noir d'or $=C_{\mathrm{c}} \simeq 0,17 \times 10^{-4} \mathrm{~J} \mathrm{~K}^{-1}$. On obtient alors, avec une seule couche d'or, une constante de temps théorique de

$$
\tau_{\text {calc }} \simeq 0,87 \mathrm{~s} \text {. }
$$

Les deux valeurs ainsi calculées encadrent la valeur moyenne mesurée $\tau_{\text {exp }}$ à $\pm 6 \%$ près.

\section{Caractéristiques comparées des thermopiles à élé- ments semiconducteurs, et à éléments métalliques.}

Si l'on remplaçait les éléments semiconducteurs par des thermocouples métalliques, la sensibilité diminuerait fortement, pour deux raisons :

- Le pouvoir thermoélectrique $\alpha_{s}$ est nettement plus faible.
- La conductivité thermique $\gamma_{i}$ est beaucoup plus forte : il sera cependant possible de réduire la conductance en utilisant des épaisseurs $e_{i}$ plus faibles.

Nous avons calculé avec le modèle précédent, la sensibilité d'une thermopile à thermocouples $\mathrm{Au}-\mathrm{Ni}$ déposés sur le même support isolant de mylar, ayant les mêmes valeurs de $r_{1}$ et $r_{2}$, le disque central et le même nombre $N$ de thermocouples. Contrairement au cas précédent (barreaux semiconducteurs), les deux éléments ont des conductivités thermiques très différentes. On peut rendre égales les conductances thermiques respectives des barreaux d'Au et de $\mathrm{Ni}$ en choisissant des épaisseurs différentes $e_{\mathrm{Au}}=e_{\mathrm{Ni}}\left(\gamma_{i} N_{i} / \gamma_{\mathrm{Au}}\right)$. On prendra par exemple comme dans [1] $=e_{\mathrm{Au}}=4 \times$ $10^{-8} \mathrm{~m}$ et $e_{\mathrm{Ni}}=12 \times 10^{-8} \mathrm{~m}$. On obtient $\sum_{i}^{\mathrm{Au}} \gamma_{i} e_{i}=$ $46 \times 10^{-7} \mathrm{~W} \mathrm{~m}^{-1} \mathrm{~K}^{-1}$ (au lieu de $8 \times 10^{-7}$ dans l'exemple précédent). D'où $\beta=0,866 \mathrm{~mm}$. La conductance thermique de la couronne $\Sigma_{\mathrm{CT}}=2 \pi r_{1} \times$ $\left(K_{\mathrm{R}} \sum_{i} \gamma_{i} e_{i}\right)^{1 / 2} F\left(r_{1}, r_{2}\right)$ est égale à $\Sigma_{\mathrm{CT}}=1,64 \times$ $10^{-4} \mathrm{~W} \mathrm{~K}^{-1}$ valeur supérieure à la conductance de rayonnement du récepteur central $\Sigma_{\mathrm{RR}}=\pi r_{1}^{2} K_{\mathrm{R}}=$ $1,11 \times 10^{-4} \mathrm{~W} \mathrm{~K}^{-1}$. (Avec les barreaux semiconducteurs, on avait $\Sigma_{\mathrm{CT}}=0,41 \times 10^{-4} \mathrm{~W} \mathrm{~K}^{-1}$, soit 4 fois moins.) La conductance totale $\Sigma_{\mathrm{T}}=\Sigma_{\mathrm{RR}}+$ $\Sigma_{\text {CT }}$ passerait de $1,52 \times 10^{-4} \mathrm{~W} \mathrm{~K}^{-1}$ à $2,75 . \times$ $10^{-4} \mathrm{~W} \mathrm{~K}^{-1}$ si on remplaçait les matériaux semiconducteurs par des barreaux d'Au et $\mathrm{Ni}$ d'épaisseur nettement plus faible.

Le coefficient de Seebeck d'un couple Au-Ni est de

$$
\alpha_{\mathrm{s}} \simeq 0,2 \times 10^{-4} \mathrm{~V} \mathrm{~K}^{-1}
$$

Par suite, une thermopile identique à la précédente, mais réalisée avec des couples $\mathrm{Au}-\mathrm{Ni}$ conduirait à :

$$
\left(S_{\mathrm{E}}\right)_{\mathrm{Au}-\mathrm{Ni}}=\frac{0,2}{2,5} \frac{1,52}{2,75}\left(S_{\mathrm{E}}\right)_{\mathrm{SC}}=4,4 \times 10^{-2}\left(S_{\mathrm{E}}\right)_{\mathrm{SC}} .
$$

Soit :

$$
\left(S_{\mathrm{E}}\right)_{\mathrm{Au}-\mathrm{Ni}} \simeq 0,6 \mathrm{~V} \mathrm{~W}^{-1} \mathrm{~cm}^{-2}
$$

La constante de temps serait elle aussi réduite :

$$
(\tau)_{\mathrm{Au}-\mathrm{Ni}} \simeq \frac{1,52}{2,75}(\tau)_{\mathrm{SC}}
$$

soit :

$$
(\tau)_{\mathrm{Au}-\mathrm{Ni}} \simeq 0,42 \mathrm{~s} .
$$

L'emploi de couples métalliques est plus favorable à une utilisation en flux modulé.

\section{Amélioration possible des caractéristiques d'une thermopile à éléments semiconducteurs.}

Pour améliorer la sensibilité, on peut accroître $\alpha_{s}$ qui, dans nos expériences n'a pu être porté à sa valeur maximum : $\left(\alpha_{\mathrm{s}} \simeq 4 \times 10^{-4} \mathrm{~V} \mathrm{~K}^{-1}\right.$ pour des matériaux massifs) par suite de l'obligation de recuire les 
films à une température maximum de $150^{\circ} \mathrm{C}$, pour éviter la destruction du mylar : cet isolant organique pourrait être remplacé par un film mince supportant des températures de recuit plus élevées.

On pourrait également accroître le nombre $N$ des thermocouples. Mais on est limité dans cette voie par l'apparition d'un bruit de fond important. Dans nos thermopiles [5] le bruit mesuré $(B \simeq 66 \times$ $10^{-9} \mathrm{~V} \mathrm{~Hz}^{-1 / 2}$, correspondant à une puissance équivalente de bruit $N E P=11 \times 10^{-10} \mathrm{~W} \mathrm{~Hz}^{-1 / 2}$ ) est dû essentiellement à la très forte résistance des thermocouples $\left(R_{\mathrm{i}} \simeq 200 \mathrm{k} \Omega\right)$. A titre de comparaison, nous avons mesuré [4] le bruit d'une thermopile commerciale RTC à éléments semi-conducteurs en $\mathrm{Te}_{2} \mathrm{Bi}_{3}$ disposés en rangées parallèles, de résistance interne $R_{\mathrm{i}} \simeq 20 \mathrm{k} \Omega$, et avons obtenu $N E P \simeq 2,6 \times$ $10^{-10} \mathrm{~W} \mathrm{~Hz}^{-1 / 2}$. Pour les thermopiles à semiconducteurs massifs décrites dans [9], où $R_{\mathrm{i}} \simeq 200 \Omega$, on aurait : $N E P \simeq 10^{-10} \mathrm{~W} \mathrm{~Hz}^{-1 / 2}$. Aucune valeur n'a été publiée pour les thermopiles à films minces métalliques $[1,2]$, mais le bruit ne doit pas y être beaucoup plus faible que dans nos thermopiles, par suite des résistances internes élevées : respectivement $R_{\mathrm{i}} \simeq 10 \mathrm{k} \Omega$ en [1] et $57 \mathrm{k} \Omega$ en [2].

Pour réduire le bruit, il faut donc diminuer la résistance interne, en accroissant l'épaisseur des rubans semiconducteurs. L'augmentation correspondante de la conductance thermique n'aurait qu'une faible influence sur la sensibilité, puisque la composante essentielle de cette conductance $\Sigma_{\mathrm{RR}}$ est due au rayonnement.

L'importance du terme $\Sigma_{\mathrm{RR}}$ a une conséquence technologique intéressante : on pourra accroître l'épaisseur de l'isolant (et donc sa conductance $\Sigma_{\mathrm{CC}}$ ) sans diminuer la sensibilité de façon significative.

La variation de $K_{\mathrm{R}}$ et de $\Sigma_{\mathrm{RR}}$ avec $T_{0}$ selon une loi en $T_{0}^{3}$ permet d'envisager une amélioration importante de la sensibilité par refroidissement - mais au détriment d'un accroissement de la constante de temps et d'une complication du fonctionnement, puisque tout l'environnement immédiat doit être porté à $T_{0}$. Une étude approfondie est nécessaire, pour chaque dispositif utilisé. En effet :

- le refroidissement accroît la conductivité thermique des matériaux constituant la couronne. Il s'agit toutefois d'une variation lente avec $T_{0}$, et la conductance radiale globale :

$$
\Sigma_{\mathrm{CT}}=\left(K_{\mathrm{R}} \sum_{i} \gamma_{i} e_{i}\right)^{1 / 2} \text { devrait diminuer avec } T_{0},
$$

- le coefficient Seebeck $\alpha_{\mathrm{S}}$ décroît avec $T_{0}$ d'un facteur 2 à 4 entre 300 et $100 \mathrm{~K}$, pour les matériaux utilisés en thermoélectricité [10],

- la résistance électrique interne $\boldsymbol{R}_{\mathrm{i}}$, pour ces matériaux, décroît très vite avec $T_{0}$, jusque vers $T_{0} \simeq 50 \mathrm{~K}$, ce qui est favorable pour la réduction du bruit.

Un accroissement de sensibilité, accompagné d'une diminution du facteur de bruit $B$ permettrait de réduire la puissance équivalente de bruit puisque :

$$
N E P\left(\mathrm{~W} \mathrm{~Hz}^{-1 / 2}\right)=\frac{B\left(\mathrm{~V} \mathrm{~Hz}^{-1 / 2}\right)}{S_{\phi}\left(\mathrm{V} \mathrm{W}^{-1}\right)}
$$

(et donc la limite inférieure du flux détectable $W_{\min }$ ) et d'accroître la détectivité :

$$
D=A_{1}^{1 / 2}(N E P)^{-1}\left(\mathrm{~W}^{-1} \mathrm{~m} \mathrm{~Hz}^{1 / 2}\right)
$$

( $A_{1}$ est l'aire du détecteur).

Pour les, thermopiles à structures radiales, objet de ce travail, avec $A_{1}=0,2 \mathrm{~cm}^{2}$, nous avons obtenu : $S_{\phi}=65 \mathrm{~V} \mathrm{~W}^{-1}, \tau=0,8 \mathrm{~s}, N E P \simeq$ $1,1 \times 10^{-9} \mathrm{~W} \mathrm{~Hz}^{-1 / 2}$ et $D=4 \times 10^{8} \mathrm{~W}^{-1} \mathrm{~cm} \mathrm{~Hz}^{1 / 2}$. Il est donc possible, grâce à un système électronique à bande passante étroite, de détecter des flux de l'ordre de $W_{\min }=10^{-9} \mathrm{~W}$, et donc d'effectuer avec une bonne précision des mesures de flux rayonné jusqu'à $W \simeq 10^{-7} \mathrm{~W}$.

Ces thermopiles ont (sans les améliorations possibles signalées plus haut) des performances comparables à celles des détecteurs pyroélectriques et des récepteurs bolométriques.

Les détecteurs pyroélectriques, associés à des résistances de charge de $10^{9}$ à $10^{12} \Omega$, ont des sensibilités $S_{\phi}$ très supérieures à celles des thermopiles $\left(10^{3}\right.$ à $5 \times 10^{3} \mathrm{~V} \mathrm{~W}^{-1}$, d'après [11] et [12]), mais le bruit $y$ est très important et finalement la puissance équivalente de bruit et la détectivité ne sont pas meilleures que celles des thermopiles :

$$
\begin{aligned}
(N E P= & 2 \times 10^{-7} \mathrm{~W} \mathrm{~Hz}^{-1 / 2} \text { en [12] et } 0,6 \mathrm{à} \\
& 2 \times 10^{-8} \mathrm{~W} \mathrm{~Hz}^{-1 / 2} \text { en [11]). }
\end{aligned}
$$

Les récepteurs bolométriques ont des performances inférieures à celles des thermopiles. Par exemple, un très bon récepteur à film mince d'or étudié par un laboratoire de Métrologie [13], d'aire utile $1 \mathrm{~cm}^{2}$, permet de détecter à la limite un flux $W_{\min } \simeq 30 \times$ $10^{-9} \mathrm{~W}$.

\section{Bibliographie}

[1] Bessonneau, C., Lebrun, J., Onde Electr. 53 (1973) 145.

[2] Studd, O., Stevens, N., Appl. Optics 7 (1968) 192.

[3] Bessonneau, C., Lebrun, J., Communication privée (1977).
[4] Balzano-Machet, C., Thèse Doctorat 3e cycle (1979) Orsay.

[5] Machet, C., Lebon, P., Septier, A., Vide Couches Minces 211 (1982) 125. 
[6] Ono, A., Metrologia 15 (1979) 127.

[7] ONo, A., Japan. J. Appl. Phys. 18 (1979) 1955.

[8] HARRIS, L., The Optical properties of metal blacks and carbon blacks, MIT and the Eppeley Foundation for Research, Monograph series no 1, 1967.

[9] Hilger-SchwarZ, Thermopiles Rank Hilger (England Technical Data Sheet $\mathrm{n}^{\circ} 3$ - 10 D/2)

[10] IofFE, A. F., Semiconductor thermoelements and Thermoelectric cooling (Edition Infosearch, London) 1958 .
[11] Putley, E. H., Infrared Phys. 20 (1980) 139.

[12] Hamilton, C. A., Day, G. W., Phelan, R. J., NBS Technical Note 678 (1976) (US Dept of Commerce, NBS, Boulder CO).

[13] Hengstberger, F., CSIR Research Report 331 (National Physical Research Laboratory, Prétoria, South Africa) 1977. 